\title{
THE IMPACT OF LOW-TAR CIGARETTES
}

\section{Bernard Stewart}

Cancer Control Program

South Eastern Sydney Public Health Unit

It seemed like a change for the best at the time. The change involved a progressive decrease, from the 1960s onwards, in the tar yield from cigarettes. The tar yield was readily measured using smoking machines. A firm basis existed for anticipating that a reduced yield of tar from cigarettes would result in a reduced incidence of lung cancer in people smoking them. So health authorities, including Cancer Councils in Australia, monitored the tar yield of cigarettes on the local market. In 1976, Wynder and Hoffman recorded that the average tar content of cigarettes in the United States fell from 31 to $24 \mathrm{mg}$ per cigarette during the period 1958-1969. ${ }^{1}$ However the prediction that smoking cigarettes with a reduced tar yield would result in a lower rate of lung cancer has not occurred. What went wrong? This article examines the development of 'low tar cigarettes', the physiology of nicotine dependence, the carcinogenic compounds contained in tobacco smoke and how these factors combine to ensure that smoking 'low tar cigarettes' does not result in a reduced risk of lung cancer.

\section{REDUCING THETAR PRODUCED BY A CIGARETTE}

The mean tar yield, as measured by smoking machine, of cigarettes sold in Australia, the United States, and other developed countries has fallen since 1960. Initially, this was due to the introduction of filter cigarettes. A filter will reduce the tar yield as recorded by a smoking machine and will also reduce the amount of tar reaching a smoker. However, subsequent to the introduction of filters, other modifications to cigarette design to reduce tar yield have been made.

To justify labelling a cigarette as 'low tar' all that is required is that the tar yield is lower than that recorded using an unmodified cigarette. The product may be then labelled 'light' or 'mild'. In the United States, since the 1970s, particular cigarette brands have been aggressively promoted on the basis of their low (machine-measured) tar; indeed, there were 'tar wars'. One way to reduce the concentration of tar reaching a smoking machine is to place tiny holes in the cigarette paper just before the filter.

Smoking is addictive because nicotine is inhaled. The nicotine yield from a cigarette, again as measured by a smoking machine, is correlated with the tar yield. It is now clear that individuals experiencing reduced nicotine intake, compensate either consciously or subconsciously by adopting certain behaviours such as smoking more cigarettes, or inhaling more frequently, or inhaling more deeply. Also, a smoker's fingers may obstruct the ventilation holes. The result is that the amount of tar reaching the smoker may be unchanged.

Hence the tar content of cigarettes refers only to the yield of tar recorded by a smoking machine. A 'low tar cigarette' is not a cigarette that results in an individual smoking receiving lesser amounts of tar than would have been the case had some other cigarette been smoked, though this may be the consumer's understanding or intention. In order to make this matter clear, the lengthy term 'cigarettes with low machine-measured yields of tar and nicotine' is now used. $^{2}$ In this article the term 'low tar cigarette' will be used to mean a cigarette with a low machine-measured yield of tar.

\section{DOESTAR MATTER?}

Lung and other cancers caused by the inhalation of tobacco smoke are attributable to two classes of chemical carcinogen: polycyclic aromatic hydrocarbons and the nitroso derivatives of nicotine and related compounds. ${ }^{3}$ Polycyclic aromatic hydrocarbons were initially recognised as the carcinogenic compounds in soot and tar, and are generated in the course of burning tobacco. These compounds are carcinogenic in experimental animals, causing a variety of cancers including lung cancer, and are present in the 'tar' component of tobacco smoke. For many years, polycyclic aromatic hydrocarbons were considered the primary carcinogens in tobacco smoke.

The nitroso derivatives of nicotine were investigated later. Nitroso derivatives of nicotine and nornicotine are present in tobacco; they are not formed as a result of combustion and account for cancer caused by chewing tobacco. These compounds cause lung cancer in experimental animals.

Hence the carcinogenic activity of tobacco smoke was initially identified with tar. Once the means were found to reduce tar yields, the opportunity was open to produce and market 'low tar cigarettes'. Against the background of low rates of cessation among young adults (specifically before the days of Nicotine Replacement Therapy), the development of 'low tar cigarettes' seemed a step in the right direction on the optimistic assumption that if the smoker must smoke then 'bad is better than worse' and 'low tar cigarettes will kill a smoker more slowly than high tar cigarettes'.

This optimism was misplaced. Two types of data provide the basis for this conclusion. First, there are physiological indicators of exposure. Blood and urinary levels of nicotine and related metabolites demonstrate that smokers of 'low tar cigarettes' do not experience a lesser dose of carcinogen. Second, consistent with exposure data, smoking 'low tar cigarettes' does not result in a reduced risk of lung cancer. 


\section{THE EXPOSURE CONSEQUENCES OF SMOKING LOWTAR CIGARETTES}

The effect of compensatory smoking behaviours has been reviewed in a monograph produced by the National Cancer Institute of the United States. ${ }^{2}$ Studies of subjects who smoked 'low tar cigarettes' support the idea that smokers regulate their intake of nicotine to sustain their addiction. Studies based on spontaneous brand switching to 'low tar cigarettes' suggest that there is no reduction in smoke intake per cigarette, and that any reductions that are seen in brand switchers depend on whether those individuals also reduce their cigarette consumption. Studies of smokers showed a weak relationship between machine-measured nicotine yield and the concentration of smokers' nicotine, carbon monoxide, or other physiological indicators.

The scenario concerning 'low tar cigarettes' suggests an 'all or nothing' maxim when it comes to the notion of modifying a smoker's exposure to tobacco-derived carcinogens. That is, there is no interim option between maintaining the habit and cessation. Even when complexities of different tar yields are set aside, the apparently reasonable presumption of reduced carcinogen exposure as a result of decreased cigarette consumption is not certain. Hecht et al. addressed the question of whether a reduction in the number of cigarettes smoked per day would decrease the carcinogen dose as indicated by urinary levels of nicotine-related metabolites. ${ }^{4}$ They recorded statistically-significant reductions in such levels caused by reductions in smoking; however, the reductions were generally modest and sometimes transient, less than 30 of 102 subjects achieved a 50 per cent decrease, and this required a reduction of 70 per cent or more in the number of cigarettes smoked.

In short, there were superficial indications that reduced levels in exposure to tobacco-derived carcinogens might be achieved by turning to 'low tar cigarettes', or even by smoking fewer cigarettes. In practice, neither of these options result in the sought-after reduction in carcinogen exposure because of other considerations, including compensatory smoking behaviours.

\section{THE DISEASE CONSEQUENCE OF SMOKING LOWTAR CIGARETTES}

Having established that usage of 'low tar cigarettes' does not achieve a commensurate reduction in carcinogen intake, public health policy must address the fallacies inherent in the marketing of 'low tar cigarettes'. Of course, changes to the marketing of cigarettes has never been accomplished on the basis of reasonable inference. Hence, we must proceed to a separate level of enquiry to answer the question: is the usage of 'low tar cigarettes' associated with reduced incidence of attributable disease, specifically lung cancer?
Results from a recent study now indicate that the inferences made from exposure data have been realised. Harris et al. compared the risk of lung cancer in smokers of medium tar cigarettes with the risk in those who smoke low tar or very low tar cigarettes. ${ }^{5}$ Compared to men who smoked medium tar cigarettes, there was no difference in the risk of lung cancer among men who smoked low tar or very low tar cigarettes. The same was seen for women. This study also found that current smokers, regardless of the tar level of their current brand of cigarettes, had substantially greater risks of lung cancer than those people who had never smoked or those who had quit smoking.

\section{THE CAUSATION OF ADENOCARCINOMA OF THE LUNG BY LOWTAR CIGARETTES}

In 1991, Devesa, Shaw and Blot initiated a registry-based study of lung cancer histology prompted by reports of a disproportionate increase in the incidence of adenocarcinoma of the lung: a scenario that they confirmed in white males that was possibly emerging in white women and also among both men and women of colour. ${ }^{6}$ The phenomenon is well established. Thun et al. prefaced their study with the observation that adenocarcinoma of the lung, once considered minimally related to cigarette smoking, has become the most common type of lung cancer in the United States, and concluded that the change seems more consistently related to changes in smoking behaviour and cigarette design than with diagnostic advances. ${ }^{7}$

The generalisation that smoking causes lung cancer is not normally qualified by reference to the principal histological subtypes of lung cancer: adenocarcinoma, squamous cell carcinoma, and small cell carcinoma. ${ }^{8}$ In brief, squamous cell carcinoma arises most frequently in the bronchi and is associated with squamous metaplasia (that is, loss of differentiated character by cells otherwise growing in sheets); adenocarcinoma tends to be peripheral in origin (arising close to the end of the bronchial tree) while small cell carcinoma is associated with a central endobrochial location.

Change in the relative incidence of adenocarcinoma is not restricted to the United States. In the Netherlands, the proportion of adenocarcinoma among men has been increasing since 1975 while survival has been decreasing; neither change is evident in women. ${ }^{9}$ Of course, as is the case everywhere, the overwhelming majority of people diagnosed with lung cancer in the Netherlands are smokers. The Dutch investigators, noting various suggestions in the literature, postulate a role for the use of filter cigarettes, which were first introduced in the Netherlands in the 1960s. Thus reduced availability of nicotine, and compensatory 'deeper' inhalation may be credibly associated with increased amounts of polycyclic hydrocarbons and nitrosated nicotine derivatives 
reaching the outer lung. It must be cautioned that simply because a hypothesis is intuitively attractive does not mean it is established..$^{10}$ However, public health action concerning the marketing and usage of low tar cigarettes need not wait upon the resolution of these mechanistic issues.

\section{CONCLUSION}

Low tar cigarettes don't work. Smoking them does not reduce the risk of lung cancer, and no basis exists for these cigarettes being recommended. On the contrary, smokers buying light or mild cigarettes have been misled and deceived if they buy these cigarettes in the hope of reducing their risk of lung cancer. This conclusion may be reached on the basis of exposure of smokers to carcinogens in tobacco smoke, and does not require documentation of the risk of disease among smokers of differing tobacco products. The case is made for a change in market practice, labelling, and the provision of health warnings.

\section{REFERENCES}

1. Wynder EL, Hoffmann D. Tobacco and tobacco smoke. Seminars in Oncology 1976; 3: 5-15.

2. National Cancer Institute. Risks associated with smoking cigarettes with low machine-measured yields of tar and nicotine. Smoking and Tobacco Control Monograph no 13.
Bethesda, MD: United States Department of Health and Human Services, National Cancer Institute, 2001.

3. Hecht SS. Tobacco carcinogens, their biomarkers and tobaccoinduced cancer. Nature Reviews Cancer 2004; 3: 733-44.

4. Hecht SS, Murphy SE, Carmella SG, Zimmerman CL, Losey L, Kramarczuk I et al. Effects of reduced cigarette smoking on the uptake of a tobacco-specific lung carcinogen. J Natl Cancer Inst 2004; 96: 107-15.

5. Harris JE, Thun MJ, Mondul AM, Calle EE. Cigarette tar yields in relation to mortality from lung cancer in the cancer prevention study II prospective cohort, 1982-88. BMJ 2004; 328: 72-80.

6. Devesa SS, Shaw GL, Blot WJ. Changing patterns of lung cancer incidence by histological type. Cancer Epidemiol Biomark Prev 2004; 1: 29-34.

7. Thun MJ, Lally CA, Flannery JT, Calle EE, Flanders WD, Heath CW. Cigarette smoking and changes in the histopathology of lung cancer. J Natl Cancer Inst 1997; 89: 1580-86.

8. Vineis P, Alavanja M, Buller P, Fontham E, Franeschi S, Gao YT et al. Tobacco and cancer: Recent epidemiological evidence. J Natl Cancer Inst 2004; 96: 99-106.

9. Janssen-Heijnen MLG, Coebergh JW, Klinkhamer PJJM, Schipper RM, Splinter TAW, Mooi WJ. Is there a common etiology for the rising incidence of and decreasing survival with adenocarcinoma of the lung? Epidemiology 2001; 12: 256-58.

10. Schuller HM. Mechanisms of smoking-related lung and pancreatic adenocarcinoma development. Nature Reviews Cancer 2002; 2: 455-63. 